\title{
NEUTRAL STAINS AS APPLIED TO THE GRANULES OF THE PANCREATIC ISLET CELLS
}

\author{
W. B. MARTIN \\ From the Anatomical Laboratory of the Johns Hopkins Medical School
}

Through the investigations of Bensley and his pupils ${ }^{1}$ we are aware that two types of cytoplasmic granules are present in the cells "composing the islets of Langerhans in the pancreas of most mammals. These granules are distinct from those found in normal pancreatic parenchyma cells and this enables us to identify islet tissue. Bensley's valuable contribution rests on the application of a neutral dye (e.g., Reinke's neutral gentian) to pancreatic tissue which has been fixed in a particular way. At the suggestion of Dr. H. M. Evans, therefore, this work was taken up with the view of determining the best method of preparation of the neutral dyes, the concentration of the staining solution necessary for the best results and further to investigate certain of the dyes allied to gentian violet and orange $G$, in the hope of obtaining a neutral stain more efficient than Reinke's neutral gentian.

Gentian violet is a mixture of two dyes of the triphenylmethane series, hexamethylpararosaniline and pentamethylpararosaniline. Therefore, when gentian violet is combined with orange $G$ the resulting neutral stain is also a mixture of two dyes and it is this mixture that is known as neutral gentian. As the two components of gentian violet differ somewhat in their staining properties and as the relative proportion of these constituents vary in different samples of gentian violet on the market it would seem advantageous to substitute the pure hexa or penta compound for the mixture. This at once suggests the substitution of other dyes of the triphenylmethane group on the basic side of the reaction and also the replacement of orange $G$ by other acid dyes of the azo series. This idea has been carried out and a number of neutral stains prepared. These dyes have been applied to pancreatic tissue fixed by the Bensley method. The result of the study of these neutral dyes is set forth below with a brief description of each dye.

The acid dyes and basic dyes combine in molecular proportion. In some cases the ratio is a simple one of $1: 1$ and in other cases the ratio may be $1: 2$ or $1: 4$. For example, ethyl violet combines with ponceau

1 M. A. Lane, The cytological characters of the areas of Langerhans. Am. Jour. Anat., vol. 7, 1907; R. R. Bensley, Studies on the pancreas of the guineapig. Am. Jour. Anat., vol. 12, 1912. 
$4 \mathrm{G} \mathrm{B}$ in the ratio of $1: 1$, with orange $\mathrm{G}$ in the ratio of $2: 1$ and with trypan blue in the ratio of $4: 1$.

The method of preparation of the neutral dye is the same in each case. A concentrated aqueous solution of the acid stain is added to a similar solution of the basic substance. This should be done slowly and the mixture stirred thoroughly. The neutral point may be determined in the following manner: After each addition of the acid substance the mixture is stirred and a drop is taken on a glass rod and placed on a piece of ordinary filter paper. The neutralized portion of the mixture, being in the form of a precipitate, settles at once on the paper, while the liquid portion containing the unneutralized stain spreads in a circle around the deposit. By the color of the outer ring can be determined whether the solution contains an excess of the acid or the basic dye, and by the change in the degree of colorization one can readily perceive the approach of the neutral point. When this is reached the outer ring is entirely colorless. The end point is thus made as exact as in any other chemical reaction and the filtrate in such a case is either clear or only slightly colored. One thus avoids an excess of either stain and the residue is practically free from either of its single constituents. This is of practical importance, for the failure to free the neutral stain of one of its components may account for some of the difficulties that have been encountered in the use of neutral gentian. The residue obtained above is filtered, washed with distilled water and allowed to dry either in the air or in an oven at a low temperature.

In order to determine the concentration of the staining solution necessary to obtain the best results, solutions of varying strength were used, the staining time remaining fixed. A solution of known strength was made up in absolute alcohol and this was kept as a stock solution. From the stock, staining solutions in 20 per cent alcohol ranging in concentration from one of $8 \mathrm{mgm}$. of the solid dye in $50 \mathrm{cc}$. of alcohol to one of $0.25 \mathrm{mgm}$. of the dye in $50 \mathrm{cc}$. alcohol were prepared. The strength of these various staining solutions is given in table 1 . Positive or negative results are indicated by the corresponding mathematical signs. To obtain the best results fairly dilute solutions should be used. A solution of neutral ethyl violet orange $G$ containing approximately $2 \mathrm{mgm}$. of the crystal dye to $50 \mathrm{cc}$. of 20 per cent alcohol is satisfactory. A staining solution of neutral azo fuchsine should contain 0.5 to $1.0 \mathrm{mgm}$. of dye to the same amount of alcohol.

When brought together in molecular proportions these dyes react with the precipitation of a neutral dye. On drying, this is a dark green powder giving a deep purple violet color in alcohol:

Crystal violet
Hydrochloride of hexamethylpararosaniline $+\left\{\begin{array}{l}\text { Orange G } \\ \text { the sodium salt of benzene- } \\ \text { azo- } \beta \text {-naphthol-disulphonic } \\ \text { acid } \gamma\end{array}\right.$ 
TABLE 1

Showing relative staining power of different neutral dyes. The numbers refer to Schultz's Farbstofftabellen, 1914

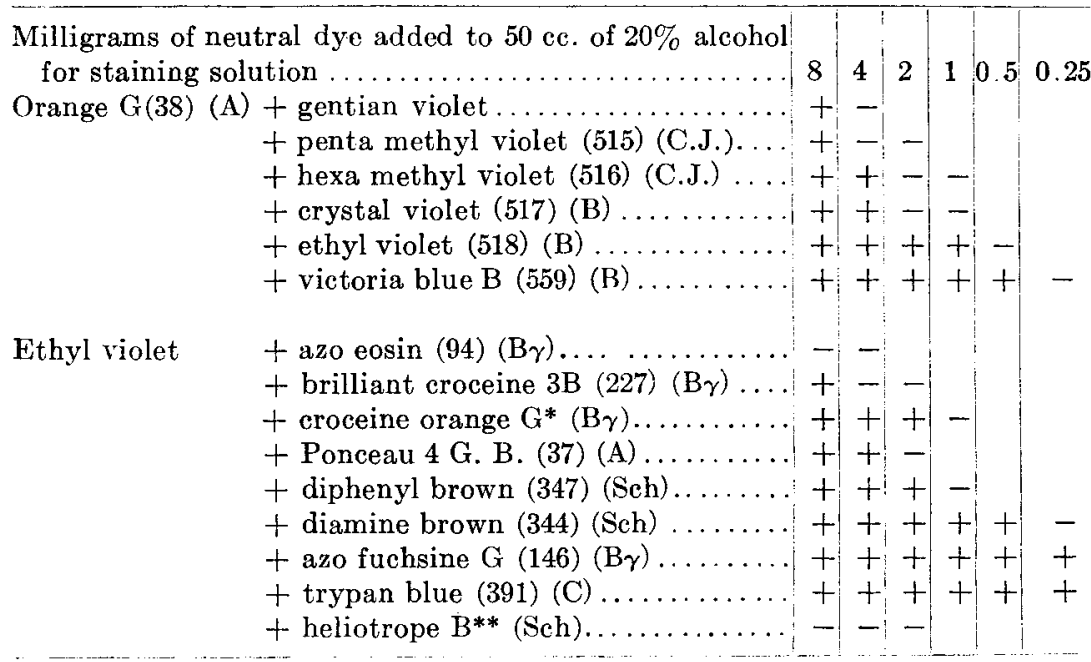

* From Fabrenfabrik of Elberteld Company. Although given by Schultz in his last edition (V) as identical with ponceau $4 \mathrm{G}$. B. the dye is undoubtedly different.

** Not given in the last edition of Schultz under this name.

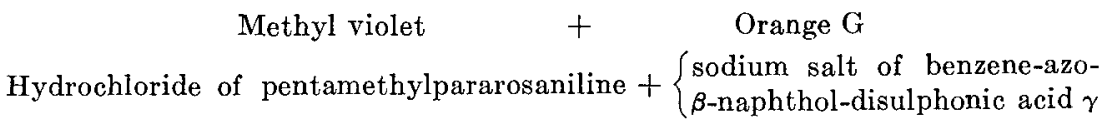

Hexa methyl violet, which is given in the table, has the same composition as crystal violet but is a purer product and gives slightly better results. Both of these dyes react with orange $\mathrm{G}$ in the same manner, giving a coarsely crystalline neutral dye of a green color and with a a fine metallic luster. Sections of pancreatic tissue stained in any one of the above dyes present much the same picture, though the hexa methyl violet is decidedly superior to the penta methyl violet. In each case the zymogen granules of the parenchyma are stained a vivid heliotrope on a light orange background. The nuclei of all the cells are blue or violet, and in the islets of Langerhans the granules in A and B cells of Lane are differentially stained in the same manner as when stained in neutral gentian. This is to be expected, as gentian violet is a mixture of methyl violet and crystal violet and in staining power lies intermediate between the two:

Ethyl violet

Hydrochloride of hexaethylpararosaniline
Orange $\mathrm{G}$ $+\left\{\begin{array}{c}\text { the sodium salt of benzene-azo- } \\ \beta \text {-naphthol-disulphonic acid } \gamma\end{array}\right.$ 
Ethyl violet and orange $G$ react in the proportion of $2: 1$ in the usual way, giving rise to a neutral stain. This forms lustrous green crystals having a slightly bronze cast and gives a violet solution in alcohol. This stain is much superior to any of the above. While the general picture is the same, it is much more intense in its action and the three types of granules are more sharply differentiated. It has the advantage also of resisting the action of the dehydrating and differentiating agents better:

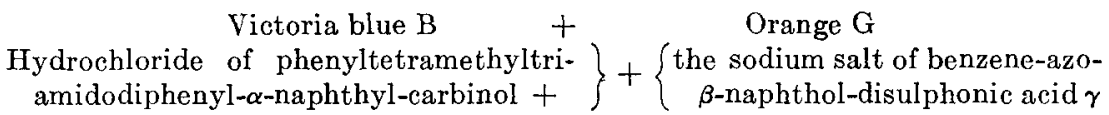

The neutral dye is obtained in the same way and forms lustrous crystals of a deep brownish-red color. The alcoholic solution is blue without the red cast of the stains so far mentioned.

Sections stained in this dye present a somewhat different picture. The zymogen granules are stained blue on a pale yellow background. The granules in the islet cells are stained a more intense blue and retain their color when differentiated from a dilute solution longer than the zymogen granules. The nuclei of all the cells are stained a beautiful blue green, the nucleolus and chromatin network standing out clearly against the rest of the nucleus. This dye will stain in high dilutions, but on account of the deficiency in color contrast between the granules and protoplasm is not as desirable a stain as the neutral ethyl violet.

The series of neutral stains so far given have been combinations of orange $\mathrm{G}$ with various basic dyes of the triphenylmethane series. Of these ethyl violet is the most valuable. In the following experiments this dye has been joined to a number of acid dyes of the azo series and the staining power of the resulting compound studied:

$$
\text { Ethyl violet _ _ Azo eosin }
$$

Hydrochloride of hexaethylpararosaniline $+\left\{\begin{array}{c}\text { the sodium salt of anisol-azo- } \alpha- \\ \text { naphthol-p-sulphonic acid }\end{array}\right.$

The neutral stain crystallizes, forming bronze green crystals, and gives a deep violet red solution in alcohol. It is, however, lacking in staining power and in concentration as high as $32 \mathrm{mgm}$. of dye to $50 \mathrm{cc}$. of 20 per cent alcohol stains the zymogen granules very faintly and the nuclei not at all:

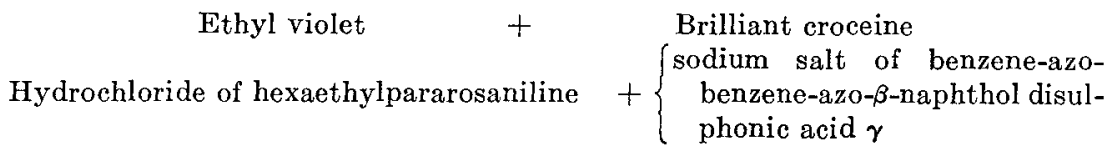

Two parts of ethyl violet combine with one part of brilliant croceine. The neutral stain is a lustrous crystalline substance of a fine green 
color forming a violet red solution in alcohol. The protoplasm of the cells stains a pale pink while the zymogen granules take a slightly darker shade. The granules in the islet cells are stained faintly and the two types can be made out but the differentiation is poor:

$$
\text { Ethyl violet } \quad+\quad \text { Croceine orange } G
$$

Fine bronze crystalline substance giving a violet red solution in alcohol. Sections stained in this dye are a bright yellow. The zymogen granules are a reddish brown. The granules of the islet cells of one type are stained a deep blue while the other type takes the same yellow color as the background. In higher dilutions the zymogen granules still take the stain but the islet granules are not stained. The nuclei are shown fairly well:

$\begin{array}{cc}\text { Ethyl violet } & + \\ \text { Hydrochloride of hexaethylpararosaniline } & +\left\{\begin{array}{c}\text { Ponceau 4 G. B. } \\ \text { sodium salt of benzene-azo- } \beta- \\ \text { naphthol- } \beta \text {-sulphonic acid }\end{array}\right.\end{array}$

These dyes combine in the proportion of $1: 1$, forming a gummy residue which crystallizes out on standing and gives a deep red solution in alcohol. The staining action of this dye is very much like that of the neutral ethyl violet orange $G$ compound except that it is dissolved out much more rapidly by differentiating agents and on this account is entirely unsatisfactory:

Hydrochloride of hexaethylpararosaniline $+\left\{\begin{array}{c}\text { Diphenyl brown } \\ \text { bedium salt of } \\ \text { benzidin } \\ \text { monomethylami- } \\ \text { donaphthol-sul- } \\ \text { phonic acid }\end{array}\right.$

Ethyl violet and diphenyl brown combine in the ratio $2: 1$ to form a neutral stain. This is a dull black amorphous powder giving a violet alcoholic solution. The zymogen granules appear heliotrope on an orange background. The granules in the islet cells take the stain but they are not sharply differentiated:

Ethyl violet $++\left\{\begin{array}{c}\text { Diamine brown } \\ \text { He sodium salt of } \\ \text { benzidin }\end{array} \quad \begin{array}{c}\text { salicylic acid } \\ \text { amidonaphtholsul- } \\ \text { phonic acid }\end{array}\right.$ 
Ethyl violet combines with diamine brown in the same proportion as with diphenyl brown to form a neutral stain. This is a dark brown powder giving a violet red solution in alcohol. Stained sections show dark red zymogen granules on a pink background. The nuclei stain exceedingly well. The granules however in the islet cells are not well differentiated and take a shade of red not very different from the background. The general picture resembles that seen in sections stained in neutral azo fuchsine except for the character of the islet granules.

Ethyl violet $++\quad \begin{gathered}\text { Heliotrope B } \\ \text { Hydrochloride of hexaethylpararosaniline }+\left\{\begin{array}{c}\text { the sodium salt of diansidine- } \\ \text { di-monoethylamidonaphtha- } \\ \text { lene sulphonic acid }\end{array}\right.\end{gathered}$

This is a dark brown amorphus powder giving a deep violet alcoholic solution. Zymogen granules are stained a light heliotrope and the protoplasm a faint pink. The granules in the islet are not well stained. This was not tried in concentrations higher than $8 \mathrm{mgm}$. of stain to 50 cc. of 20 per cent alcohol and since it offers a fairly good color contrast it might be useful in higher concentrations.

Ethyl violet
Hydrochloride of hexaethylpararosanaline $+\left\{\begin{array}{l}\text { Trypan blue } \\ \text { the sodium salt of tolidine- } \\ \text { diamidonaphtholdisulphonic } \\ \text { acid } H\end{array}\right.$

Four parts of ethyl violet are required to neutralize one part of trypan blue. The neutral dye is a deep green crystalline substance. The alcoholic solution is blue with a slight violet tint. This is a very intense stain and resists the action of alcohol and acetone. The zymogen granules are stained a deep purple blue against a light blue background. The nuclei stain fairly well. The differentiation of the granules in the islet cells is however poor. Both types appear to take the stain but the color contrast throughout is not sharp enough:

$\begin{gathered}\text { Ethyl violet } \\ \text { Hydrochloride of hexaethylpararosaniline }\end{gathered}+\left\{\begin{array}{l}\text { Azo fuchsine } \\ \text { the sodium salt of p-sulphoben- } \\ \text { zene-azo-dioxynaphthyalene } \\ \text { sulphonic acid }\end{array}\right.$

Ethyl violet and azo fuchsine combine in the ratio of $2: 1$. The dry neutral dye prepared in the same way as the above is a fine crystalline powder of a green color which gives a violet red solution in alcohol. Pancreatic tissue stained in this dye presents a very brilliant picture. The zymogen granules are a deep purple on a light pink background. The nuclei in all the cells are stained fairly well, the chromatin material being red. The two types of granules in the islet cells are stained differentially, one type taking a violet stain and the other a distinct red. This is a very intense stain and may be used in high dilutions. 
It is resistant to the action of acetone and alcohol thus making possible a more careful differentiation than with any of the other stains used. Sections stained in it have little tendency to fade and preparations made nearly a year ago are as brilliant as when first made.

From a consideration of the group of dyes just described it is evident that two of them stand out as distinctly superior to any of the others as a stain for pancreatic tissue. These are the compounds formed by the union of ethyl violet with orange $G$ and with azo fuchsine. These are both powerful dyes, staining cell granules very intensely. The color contrast between the different types of granules and between the granules and the cell protoplasm is very sharp. They are efficient in high dilutions and gross precipitation of stain on the tissue is avoided. Finally it may be said the granules and the nuclear chromatin retain these stains well in the presence of acetone and absolute alcohol, thus rendering differentiation easy.

On referring to the table given above it is seen that the neutral stains formed by combining orange $G$ with different basic dyes vary in staining strength and that as the basic substance used becomes more complexed the staining power of the neutral dye increases. Thus, the hexamethyl violet gives a more efficient stain than the penta methyl violet, the hexa ethyl compound surpasses the hexa methyl combinations while victoria blue joined to orange $G$ gives a neutral dye that will stain efficiently in higher dilutions t: an any of the others

The dyes tested in the above study were not secured through dealers but in each instance from the firm concerned in its manufacture. I am indebted to Dr. Evans, who placed his collection at my disposal, and we wish to thank the following houses for coöperation both in the supply of dye samples and in the confirmation of the precise chemical make-up of the dyes used: Farbenfabrike of Elberfeld Company ; the Badische Company; the Berline-Aniline Works; Kalle and Company; Leopold Cassella \& Co.; Carl Jäger; and Schoellkoff, Hartford \& Hanna Co. 